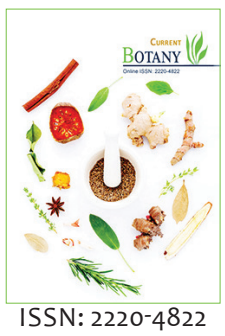

Received: June 20, 2020 Revised: August 17, 2020 Accepted: August 25, 2020 Published: August 30, 2020

*Corresponding Author: Riyad Hossen

E-mail: rhossen@bu.ac.bd

\section{First report on phytoplankton communities of Barishal City, Bangladesh}

\author{
Shaswati Chakraborty, Dipalok Karmaker, Subroto Kumar Das and \\ Riyad Hossen*
}

Department of Botany, University of Barishal, Barishal-8200, Bangladesh

\begin{abstract}
Phytoplanktons, also called microalgae, are microscopic photosynthetic living organisms that generally found in aquatic environments. Although they are considered as the most important primary producers and bioindicators of aquatic ecosystems, there was no previous report found for Barishal City about these tiny organisms. Consequently, the present study selected 10 freshwater reservoirs from the city to investigate phytoplankton communities and listed 110 taxa under 4 phyla, 7 classes, 18 orders, 24 families and 49 genera. The distribution of Chlorophytes was abundant relatively in terms of species number ( 45 taxa) followed by Euglenophytes, Chlorophytes and Cyanophytes in this area. Only Euglenaceae possessed one-third of the total species of this report. Among all stations, the highest number of taxa was recorded from station 2 and according to nine biodiversity indices, the station 2 and 9 showed comparatively good results. All of the recorded taxa were previously mentioned by different authors from Bangladesh.
\end{abstract}

KEYWORDS: Phytoplankton, Microalgae, Scenedesmus abundans, Barishal and Bangladesh

\section{INTRODUCTION}

Algae are considered as sole primary producers in oceans [1] and one of the most important primary producers in freshwater ecosystems. The term 'phytoplankton' also called microalgae is generally referred to mean microscopic algae to cyanobacteria, and they provide a major share of oxygen in an aquatic ecosystem. Besides, they serve as foods, fertilizers and considered as an effective bio-indicator for fishing as well as assessing water quality. Furthermore, several bioactive compounds have been extracted from phytoplankton, which have the properties of antioxidant, anti-inflammatory, anticancer, and antiviral medicines [2]. Thus, phytoplanktons have been considered as an alternate of synthetic dietary supplements for treatments of many human diseases [3]. And for their high lipid content per cell, rapid growth rate, biodegradable, renewable and environment-friendly natures, they have been regarded as a prospective source of biofuel to reduce the use of terrestrial food crops for biofuel production in future [4].

Barishal is one of the oldest beautiful municipal with a large number of freshwater reservoirs and the second largest river ports of Bangladesh. The City is located in the southern part of this country and lies on the bank of Kirtankhola River. The area of the City is $24.91 \mathrm{~km}^{2}$ located in between $22^{\circ} 38^{\prime}$ and $22^{\circ} 45^{\prime}$ north latitudes as well as $90^{\circ} 18^{\prime}$ and $90^{\circ} 23^{\prime}$ east longitudes [5]. As the City is expanding, several industries are operating already near to many ponds or lakes and thus the water is being polluted by waste dispersal and leakages. To assess the water quality of the area, phytoplankton would be the most important bio-indicators and sometimes they would be far better than other parameters. Moreover, to measure biodiversity of any region phytoplankton must be included as a large group of aquatic microorganisms. Some previous investigations were done on the phytoplankton communities from Barishal divisional region, such as Pirojpur district [6] and Bakerganj upazila of Barishal district [7]. But there were no available reports found on phytoplankton communities of Barishal City.

Diversity of freshwater phytoplanktons is highly complex in an aquatic environment because diversity consists of two components, the variety and the relative abundance of species. Even ecologists set many indices to measure diversity and it is obviously an important tool for measuring the species status of an area. Therefore, the main goal of this work was recording phytoplankton species of Barishal City with their distribution and diversity. Moreover, outcome of the study would be helpful 
to analyze the water quality, environment pollutions, and biodiversity of this region.

\section{MATERIALS AND METHODS}

\section{Study Area}

The survey was carried out between September 2019 and January 2020 from 10 stations (St.) of Barishal City (Figure 1). The stations were Rupatali Pond (1), Rupatali Lake (2), DC Office Pond (3), DC Lake (4), Gol Pukur (5). Kalushah Sarak Pond (6), Kawnia Road Pond (7), Notun Bazar Pond (8), College Road Pond (9) and Nazrul Islam Sarak Pond (10).

\section{Samples Collection}

Samples (1L water) were collected between 7 to 10 am from each station. They were collected from the surface layer of 10 to $50 \mathrm{~cm}$ depth with Ruttner water sampler and fixed with $4 \%$ neutral formalin before transferring to graduated cylinders (1L capacity). Then added a few drops of Lugol's solution and left for 48 hours to sediment. The supernatant water was then siphoned until the sample was concentrated to $100 \mathrm{ml}$. Finally, the sediment was examined under a light microscope (100x magnification) equipped with digital camera for photographing, recording and measuring.

\section{Taxonomy \& Identification}

Identification and enumeration were done by a binocular microscope. And as literatures, Bellinger and Sigee [8],

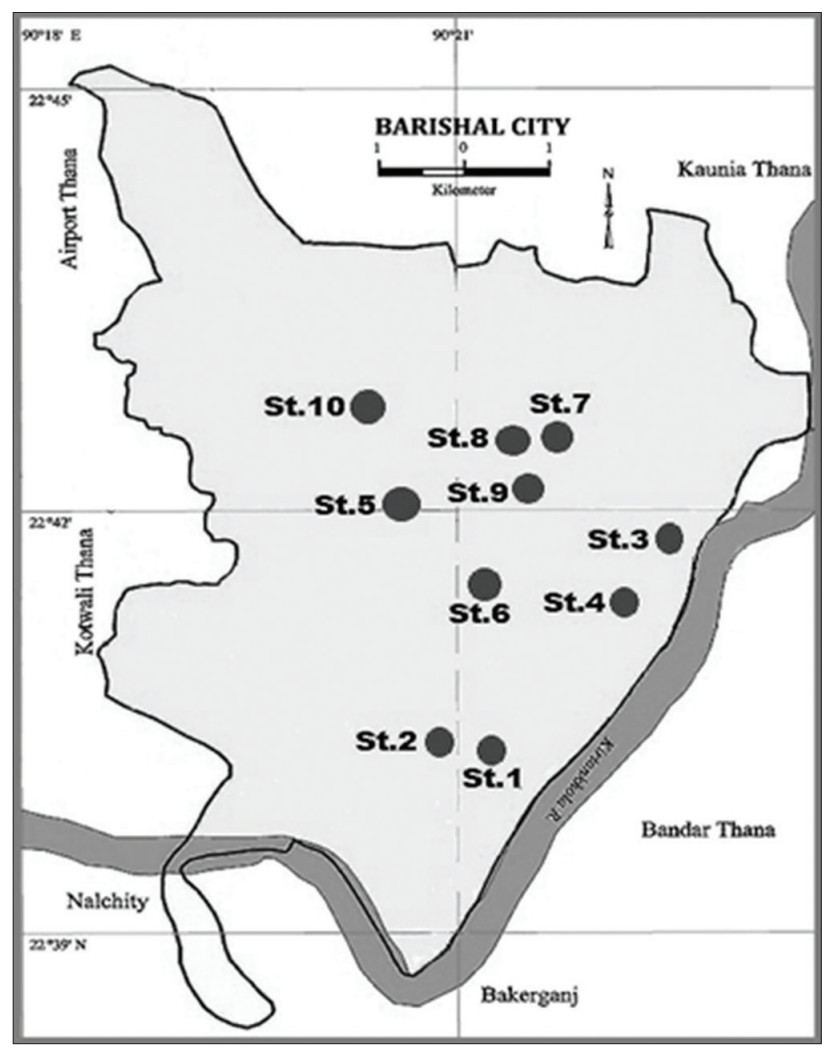

Figure 1: Barishal City map showing all sampling stations
Ahmed et al. [9], Islam and Alfasane [10], Islam and Moniruzzaman [11], and Smith [12] were followed to confirm identification. Moreover, the presented taxonomic arrangements and classifications were prepared based on Robert Edward Lee [13], but in some special cases Komárek and Fott [14], and Bold and Wynne [15] were consulted.

\section{Distribution \& Diversity Measurement}

The frequency was counted by using heamocytometer based on the percent occurrence of an individual species to refer species distribution. The rare and the dominant species were indicated following the resulted frequency. The phytoplanktons were expressed as organisms per $\mathrm{ml}$ for the purpose of calculating diversity indices and the data were subjected to a software program PAST which generates nine diversity indices (Dominance index, Shannon index, Simpson index, Pielou's index, Menchinick's index, Margalef's index, Equitability index, Fisher alpha index and Berger-Parker's Dominance Index).

\section{RESULTS}

A total of 110 taxa including 16 prokaryotic and 94 eukaryotic phytoplanktons were recorded from the City. They were found belonging to the four major phyla Cyanophyta, Chlorophyta, Heterokontophyta and Euglenophyta within 49 genera, 24 families, 18 orders and 7 classes. The Chlorophytes were found dominantly in terms of the percentage of taxa present in the study (41\%), while the Cyanophytes and Heterokontophytes were less dominant comparatively (Figure 2). All taxa of the survey were listed in the table 1 with their brief description and distribution. Then the taxonomic classifications were presented in the table 2 . The classifications were arranged following alphabetic orders and all prokaryotes were presented first following the eukaryotes. The habits found in the study were colonial, filamentous, aggregated, coenobial and solitary. The listed phytoplanktons were spherical, oval, square, round, conical, disk, curved, crescent, spindle, elliptical, leaf, triangular, drum, boat, needle, horn, linear, and fusiform shaped. And, their cell size ranges from $1.5 \times 2$ to $21 \times 95 \mu \mathrm{m}$.

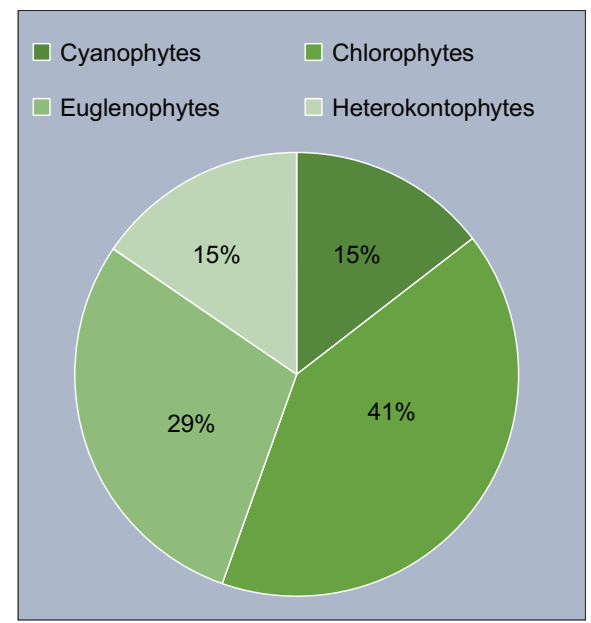

Figure 2: Relative distributions of phytoplanktons under four phyla 
Table 1:List of phytoplanktons found in the 10 stations of Barishal City with their brief description and distribution

\begin{tabular}{|c|c|c|c|c|c|c|c|c|c|c|c|c|c|c|}
\hline \multirow[t]{2}{*}{ No. } & \multirow[t]{2}{*}{ Name } & \multirow[t]{2}{*}{ Habit (Cell) } & \multirow[t]{2}{*}{ Shape (Cell) } & \multirow[t]{2}{*}{ Size $(\mu \mathrm{m})$} & \multicolumn{10}{|c|}{ Distribution (Frequency) } \\
\hline & & & & & St.1 & St.2 & St.3 & St.4 & St.5 & St.6 & St.7 & St.8 & St.9 & St.10 \\
\hline 1 & Chroococcus dispersus & Colonial & Spherical & $3 \times 4.5$ & 1 & 0 & 0 & 0 & 0 & 1 & 0 & 0 & 2 & 0 \\
\hline 2 & Chroococcus minor & Colonial & Spherical & $3.5 \times 4$ & 0 & 0 & 0 & 2 & 1 & 0 & 0 & 0 & 0 & 15 \\
\hline 3 & Gloeothece rupestris & Colonial & Oval & $4 \times 5.5$ & 2 & 2 & 0 & 0 & 0 & 13 & 1 & 3 & 0 & 11 \\
\hline 4 & Merismopedia angularis & Colonial & Square & $5 \times 5.5$ & 0 & 0 & 1 & 0 & 0 & 17 & 0 & 0 & 0 & 1 \\
\hline 5 & Merismopedia glauca & Colonial & Square & $2 \times 3.5$ & 0 & 0 & 0 & 0 & 0 & 10 & 12 & 0 & 0 & 0 \\
\hline 6 & Merismopedia punctata & Colonial & Square & $3 \times 3.5$ & 0 & 2 & 0 & 8 & 8 & 0 & 1 & 10 & 0 & 0 \\
\hline 7 & Merismopedia trolleri & Colonial & Square & $4 \times 5.5$ & 2 & 0 & 0 & 0 & 9 & 0 & 2 & 1 & 0 & 9 \\
\hline 8 & Microcystis aeruginosa & Colonial & Round & $2.5 \times 3$ & 8 & 7 & 8 & 0 & 0 & 1 & 0 & 0 & 2 & 2 \\
\hline 9 & Microcystis densa & Colonial & Round & $2.5 \times 3$ & 2 & 0 & 0 & 2 & 0 & 11 & 16 & 0 & 0 & 3 \\
\hline 10 & Microcystis flosaquae & Colonial & Oval & $2.5 \times 3$ & 0 & 2 & 2 & 1 & 8 & 0 & 2 & 9 & 0 & 7 \\
\hline 11 & Synechocystis aquatilis & Solitary & Oval & $3.5 \times 5$ & 7 & 0 & 0 & 0 & 0 & 0 & 0 & 0 & 0 & 0 \\
\hline 12 & Anabaena raciborskii & Filament & Conical & $6 \times 10$ & 0 & 0 & 12 & 2 & 0 & 0 & 0 & 0 & 0 & 0 \\
\hline 13 & Anabaena volzii & Filament & Round & $6 \times 9.5$ & 0 & 0 & 8 & 1 & 0 & 0 & 0 & 0 & 0 & 0 \\
\hline 14 & Calothrix scytonemicola & Filament & Spherical & $6.5 \times 8$ & 0 & 0 & 13 & 15 & 0 & 0 & 0 & 0 & 0 & 1 \\
\hline 15 & Oscillatoria formosa & Filament & Round & $3.5 \times 4$ & 6 & 6 & 9 & 18 & 0 & 0 & 0 & 2 & 2 & 0 \\
\hline 16 & Spirulina major & Filament & Disk & $6 \times 8.5$ & 0 & 0 & 2 & 0 & 0 & 2 & 0 & 0 & 0 & 0 \\
\hline 17 & Characium limneticum & Solitary & Oval & $7.5 \times 9$ & 0 & 1 & 0 & 1 & 0 & 8 & 8 & 7 & 0 & 0 \\
\hline 18 & Characium rostratum & Solitary & Spindle & $8.5 \times 12$ & 0 & 0 & 0 & 0 & 0 & 0 & 0 & 1 & 0 & 3 \\
\hline 19 & Closterium dianae & Solitary & Curved & $16 \times 90$ & 0 & 7 & 0 & 0 & 0 & 0 & 7 & 0 & 0 & 6 \\
\hline 20 & Closterium incurvum & Solitary & Crescent & $18 \times 85$ & 0 & 0 & 0 & 0 & 1 & 0 & 0 & 0 & 0 & 1 \\
\hline 21 & Closterium kuetzingii & Solitary & Curved & $20 \times 90$ & 2 & 0 & 0 & 0 & 0 & 0 & 2 & 0 & 0 & 0 \\
\hline 22 & Closterium nematodes & Aggregated & Crescent & $13 \times 95$ & 0 & 5 & 0 & 0 & 6 & 0 & 0 & 0 & 0 & 0 \\
\hline 23 & Closterium setaceum & Solitary & Curved & $12 \times 90$ & 0 & 1 & 0 & 0 & 0 & 1 & 11 & 9 & 1 & 0 \\
\hline 24 & Closterium subulatum & Solitary & Crescent & $21 \times 95$ & 1 & 0 & 0 & 0 & 0 & 0 & 0 & 1 & 0 & 0 \\
\hline 25 & Actinotaenium turgidum & Solitary & Fusiform & $14 \times 20$ & 0 & 0 & 0 & 0 & 0 & 0 & 0 & 2 & 2 & 1 \\
\hline 26 & Cosmarium moniliforme & Solitary & Oval & $17 \times 19$ & 0 & 0 & 0 & 2 & 0 & 0 & 0 & 0 & 0 & 2 \\
\hline 27 & Cosmarium portianum & Filament & Round & $20 \times 22$ & 0 & 0 & 1 & 1 & 0 & 0 & 0 & 7 & 0 & 7 \\
\hline 28 & Cosmarium tumidum & Solitary & Round & $17 \times 19$ & 9 & 0 & 2 & 1 & 0 & 0 & 0 & 0 & 0 & 8 \\
\hline 29 & Euastrum elegans & Solitary & Round & $3 \times 4.5$ & 0 & 7 & 0 & 0 & 0 & 0 & 0 & 0 & 0 & 0 \\
\hline 30 & Teilingia exigua & Solitary & Elliptical & $1.5 \times 2$ & 0 & 0 & 2 & 0 & 0 & 0 & 0 & 0 & 0 & 0 \\
\hline 31 & Chaetopeltis orbicularis & Aggregated & Oval & $10 \times 13$ & 0 & 0 & 0 & 7 & 1 & 0 & 6 & 1 & 0 & 0 \\
\hline 32 & Chlorella vulgaris & Solitary & Elliptical & $2 \times 2.5$ & 0 & 0 & 0 & 0 & 0 & 0 & 0 & 0 & 0 & 13 \\
\hline 33 & Chlorococcum infusionum & Solitary & Elliptical & $9.0 \times 40$ & 1 & 0 & 0 & 0 & 5 & 5 & 0 & 0 & 0 & 0 \\
\hline 34 & Hyaloraphidium contortum & Solitary & Curved & $3 \times 5.5$ & 0 & 2 & 0 & 0 & 0 & 0 & 0 & 0 & 0 & 2 \\
\hline 35 & Schroederia setigera & Solitary & Spindle & $12.5 \times 5$ & 1 & 0 & 0 & 0 & 0 & 0 & 0 & 0 & 0 & 8 \\
\hline 36 & Tetraedron minimum & Solitary & Triangular & $9.5 \times 12$ & 0 & 0 & 2 & 1 & 0 & 0 & 0 & 0 & 5 & 0 \\
\hline 37 & Closteriopsis longissima & Solitary & Crescent & $7.5 \times 75$ & 0 & 0 & 0 & 0 & 0 & 6 & 3 & 0 & 0 & 0 \\
\hline 38 & Oocystis lacustris & Coenobial & Oval & $9 \times 9.5$ & 1 & 1 & 0 & 0 & 4 & 0 & 0 & 0 & 0 & 0 \\
\hline 39 & Oocystis submarina & Solitary & Fusiform & $11 \times 17$ & 1 & 2 & 0 & 0 & 0 & 0 & 0 & 0 & 0 & 0 \\
\hline 40 & Planktosphaeria gelatinosa & Solitary & Oval & $4 \times 4.5$ & 0 & 0 & 0 & 0 & 3 & 0 & 0 & 0 & 0 & 0 \\
\hline 41 & Pediastrum duplex & Coenobial & Horn & $8.0 \times 30$ & 0 & 0 & 0 & 3 & 0 & 0 & 5 & 0 & 0 & 0 \\
\hline 42 & Actinastrum hantzschii & Coenobial & Linear & $2.5 \times 7$ & 0 & 1 & 0 & 0 & 0 & 0 & 0 & 0 & 1 & 5 \\
\hline 43 & Scenedesmus abundans & Coenobial & Round & $4 \times 8.5$ & 0 & 0 & 0 & 0 & 0 & 11 & 13 & 3 & 0 & 2 \\
\hline 44 & Crucigenia crucifera & Coenobial & Oval & $5.5 \times 16$ & 0 & 5 & 1 & 1 & 0 & 0 & 0 & 0 & 0 & 0 \\
\hline 45 & Crucigenia tetrapedia & Coenobial & Crescent & $6.5 \times 15$ & 0 & 0 & 5 & 5 & 0 & 2 & 0 & 0 & 0 & 0 \\
\hline 46 & Coelastrum microporum & Coenobial & Spherical & $8.0 \times 12$ & 4 & 1 & 4 & 0 & 0 & 0 & 0 & 1 & 0 & 1 \\
\hline 47 & Kirchneriella contorta & Coenobial & Linear & $1.5 \times 12$ & 4 & 1 & 5 & 4 & 0 & 0 & 0 & 1 & 1 & 0 \\
\hline 48 & Scenedesmus longispina & Coenobial & Fusiform & $6 \times 7.5$ & 0 & 0 & 0 & 0 & 0 & 0 & 0 & 0 & 1 & 2 \\
\hline 49 & Scenedesmus quadricauda & Coenobial & Spherical & $8.5 \times 12$ & 5 & 6 & 0 & 0 & 0 & 1 & 0 & 0 & 1 & 1 \\
\hline 50 & Scenedesmus regularis & Coenobial & Elliptical & $6.5 \times 12$ & 0 & 7 & 2 & 0 & 0 & 0 & 9 & 0 & 1 & 0 \\
\hline 51 & Scenedesmus acuminatus & Colonial & Needle & $2.5 \times 11$ & 0 & 0 & 0 & 0 & 0 & 0 & 2 & 0 & 0 & 0 \\
\hline 52 & Pandorina morum & Colonial & Crescent & $3.5 \times 12$ & 5 & 0 & 0 & 0 & 0 & 0 & 0 & 0 & 0 & 0 \\
\hline 53 & Korshikoviella limnetica & Solitary & Linear & $2 \times 8.0$ & 0 & 0 & 0 & 4 & 0 & 0 & 0 & 0 & 0 & 0 \\
\hline 54 & Asterococcus limneticus & Colonial & Oval & $7.5 \times 8$ & 0 & 0 & 0 & 0 & 0 & 1 & 1 & 0 & 0 & 4 \\
\hline 55 & Gloeocystis vesiculosa & Aggregated & Round & $6.5 \times 8$ & 0 & 5 & 0 & 0 & 0 & 2 & 0 & 0 & 0 & 2 \\
\hline 56 & Chlamydomonas acidophila & Solitary & Fusiform & $7 \times 9.5$ & 0 & 0 & 2 & 0 & 3 & 0 & 0 & 0 & 0 & 1 \\
\hline 57 & Chlamydomonas angulosa & Solitary & Spherical & $6 \times 8.5$ & 0 & 0 & 0 & 0 & 1 & 4 & 4 & 0 & 0 & 0 \\
\hline 58 & Chlamydomonas botryopara & Solitary & Spherical & $7.0 \times 10$ & 0 & 0 & 1 & 2 & 0 & 0 & 0 & 0 & 0 & 0 \\
\hline 59 & Chlamydomonas globosa & Solitary & Oval & $4 \times 6.5$ & 5 & 0 & 1 & 1 & 0 & 0 & 6 & 0 & 0 & 0 \\
\hline 60 & Eudorina elegans & Colonial & Spherical & $8.5 \times 10$ & 0 & 0 & 0 & 0 & 1 & 0 & 0 & 0 & 1 & 4 \\
\hline 61 & Kirchneriella irregularis & Colonial & Oval & $4 \times 6.5$ & 1 & 1 & 0 & 0 & 0 & 0 & 0 & 0 & 0 & 0 \\
\hline 62 & Euglena acus & Solitary & Spindle & $5.5 \times 17$ & 2 & 0 & 0 & 0 & 1 & 0 & 0 & 0 & 0 & 0 \\
\hline 63 & Euglena chlamydophora & Solitary & Spindle & $5.5 \times 17$ & 1 & 0 & 1 & 0 & 0 & 0 & 0 & 2 & 0 & 1 \\
\hline 64 & Euglena clavata & Solitary & Spindle & $5.0 \times 16$ & 0 & 0 & 2 & 2 & 0 & 0 & 0 & 0 & 0 & 0 \\
\hline 65 & Euglena flava & Solitary & Spindle & $4.5 \times 14$ & 18 & 0 & 0 & 0 & 0 & 0 & 0 & 14 & 0 & 0 \\
\hline
\end{tabular}


Table 1: (Continued)

\begin{tabular}{|c|c|c|c|c|c|c|c|c|c|c|c|c|c|c|}
\hline \multirow[t]{2}{*}{ No. } & \multirow[t]{2}{*}{ Name } & \multirow[t]{2}{*}{ Habit (Cell) } & \multirow[t]{2}{*}{ Shape (Cell) } & \multirow[t]{2}{*}{ Size $(\mu \mathrm{m})$} & \multicolumn{10}{|c|}{ Distribution (Frequency) } \\
\hline & & & & & St.1 & St.2 & St.3 & St.4 & St.5 & St.6 & St.7 & St.8 & St.9 & St.10 \\
\hline 66 & Euglena geniculata & Solitary & Spindle & $4.0 \times 15$ & 0 & 11 & 0 & 0 & 2 & 10 & 0 & 3 & 0 & 0 \\
\hline 67 & Euglena granulata & Solitary & Spindle & $4.0 \times 14$ & 0 & 0 & 0 & 0 & 0 & 0 & 0 & 0 & 2 & 0 \\
\hline 68 & Euglena pisciformis & Solitary & Spindle & $4.5 \times 65$ & 0 & 0 & 0 & 0 & 0 & 0 & 0 & 3 & 1 & 0 \\
\hline 69 & Euglena polymorpha & Solitary & Spindle & $8.5 \times 75$ & 0 & 1 & 1 & 2 & 0 & 0 & 6 & 0 & 0 & 0 \\
\hline 70 & Euglena proxima & Solitary & Curved & $11 \times 55$ & 5 & 5 & 0 & 1 & 1 & 0 & 0 & 0 & 0 & 0 \\
\hline 71 & Euglena sociabilis & Solitary & Spindle & $7.0 \times 75$ & 1 & 0 & 0 & 0 & 0 & 1 & 1 & 0 & 0 & 7 \\
\hline 72 & Euglena spirogyra & Solitary & Curved & $12 \times 70$ & 0 & 1 & 4 & 5 & 0 & 0 & 0 & 1 & 0 & 0 \\
\hline 73 & Euglena tripteris & Solitary & Spindle & $11 \times 65$ & 0 & 0 & 0 & 0 & 0 & 0 & 6 & 0 & 2 & 2 \\
\hline 74 & Euglena variabilis & Solitary & Oval & $21 \times 77$ & 0 & 1 & 2 & 1 & 0 & 0 & 0 & 2 & 0 & 0 \\
\hline 75 & Lepocinclis acuta & Solitary & Oval & $8 \times 9.5$ & 0 & 4 & 1 & 0 & 0 & 0 & 0 & 0 & 1 & 0 \\
\hline 76 & Lepocinclis ovum & Solitary & Spherical & $14 \times 19$ & 0 & 0 & 0 & 0 & 8 & 0 & 0 & 0 & 1 & 0 \\
\hline 77 & Lepocinclis playfairiana & Solitary & Spherical & $15 \times 19$ & 0 & 0 & 0 & 2 & 0 & 0 & 0 & 0 & 0 & 3 \\
\hline 78 & Lepocinclis sphagnophila & Solitary & Spindle & $7 \times 8.5$ & 0 & 0 & 0 & 0 & 0 & 3 & 0 & 0 & 0 & 0 \\
\hline 79 & Lepocinclis teres & Solitary & Oval & $7.5 \times 9$ & 1 & 1 & 0 & 5 & 0 & 0 & 0 & 0 & 0 & 4 \\
\hline 80 & Lepocinclis texta & Solitary & Oval & $14 \times 35$ & 0 & 0 & 1 & 1 & 0 & 0 & 0 & 0 & 0 & 4 \\
\hline 81 & Phacus acuminatus & Solitary & Leaf & $30 \times 40$ & 0 & 0 & 4 & 0 & 0 & 0 & 0 & 0 & 0 & 0 \\
\hline 82 & Phacus caudatus & Solitary & Leaf & $11 \times 25$ & 0 & 0 & 0 & 0 & 1 & 0 & 0 & 1 & 2 & 0 \\
\hline 83 & Phacus curvicauda & Solitary & Leaf & $35 \times 65$ & 0 & 2 & 0 & 0 & 2 & 1 & 0 & 0 & 0 & 0 \\
\hline 84 & Phacus denisii & Solitary & Leaf & $30 \times 40$ & 0 & 0 & 0 & 0 & 1 & 2 & 0 & 0 & 0 & 0 \\
\hline 85 & Phacus hamatus & Solitary & Oval & $9.0 \times 15$ & 0 & 0 & 4 & 5 & 0 & 0 & 0 & 0 & 0 & 1 \\
\hline 86 & Phacus pseudonordstedii & Solitary & Oval & $11 \times 19$ & 0 & 2 & 0 & 0 & 1 & 0 & 0 & 2 & 0 & 0 \\
\hline 87 & Strombomonas gibberosa & Solitary & Oval & $15 \times 19$ & 1 & 0 & 0 & 0 & 4 & 1 & 0 & 0 & 0 & 0 \\
\hline 88 & Trachelomonas granulosa & Solitary & Spherical & $11 \times 18$ & 0 & 0 & 0 & 0 & 5 & 0 & 3 & 0 & 0 & 1 \\
\hline 89 & Trachelomonas hispida & Solitary & Spherical & $18 \times 28$ & 0 & 0 & 0 & 0 & 4 & 0 & 0 & 0 & 0 & 0 \\
\hline 90 & Trachelomonas oblonga & Solitary & Elliptical & $7.5 \times 16$ & 0 & 3 & 0 & 0 & 1 & 0 & 0 & 0 & 0 & 0 \\
\hline 91 & Trachelomonas pulcherrima & Solitary & Spherical & $10 \times 20$ & 0 & 0 & 0 & 0 & 1 & 2 & 0 & 0 & 0 & 6 \\
\hline 92 & Trachelomonas pusilla & Solitary & Elliptical & $11 \times 14$ & 0 & 0 & 1 & 2 & 0 & 1 & 0 & 0 & 0 & 0 \\
\hline 93 & Trachelomonas robusta & Solitary & Spherical & $21 \times 29$ & 0 & 1 & 0 & 1 & 0 & 0 & 0 & 0 & 0 & 0 \\
\hline 94 & Melosira granulata & Colonial & Spherical & $8.0 \times 14$ & 0 & 8 & 5 & 0 & 0 & 0 & 0 & 0 & 0 & 0 \\
\hline 95 & Melosira varians & Colonial & Spherical & $10 \times 21$ & 3 & 0 & 0 & 0 & 0 & 3 & 0 & 0 & 0 & 0 \\
\hline 96 & Gomphonema lanceolatum & Solitary & Leaf & $13 \times 45$ & 0 & 0 & 1 & 1 & 0 & 4 & 0 & 0 & 0 & 0 \\
\hline 97 & Gomphonema subtile & Solitary & Leaf & $13 \times 37$ & 1 & 0 & 0 & 0 & 0 & 4 & 4 & 0 & 1 & 1 \\
\hline 98 & Nitzschia acicularis & Solitary & Needle & $5.0 \times 40$ & 0 & 5 & 0 & 0 & 5 & 0 & 0 & 0 & 0 & 0 \\
\hline 99 & Nitzschia longissima & Solitary & Needle & $4.5 \times 30$ & 0 & 0 & 0 & 3 & 0 & 0 & 0 & 3 & 0 & 0 \\
\hline 100 & Navicula cuspidata & Solitary & Boat & $21 \times 60$ & 0 & 0 & 0 & 0 & 0 & 0 & 2 & 0 & 0 & 0 \\
\hline 101 & Navicula exigua & Solitary & Elliptical & $7.5 \times 21$ & 0 & 3 & 0 & 0 & 0 & 0 & 0 & 0 & 2 & 0 \\
\hline 102 & Navicula menisculus & Solitary & Boat & $6.5 \times 26$ & 0 & 0 & 0 & 0 & 0 & 0 & 0 & 0 & 9 & 0 \\
\hline 103 & Pinnularia acrosphaeria & Solitary & Boat & $11 \times 82$ & 6 & 0 & 6 & 0 & 0 & 2 & 0 & 0 & 1 & 0 \\
\hline 104 & Pinnularia acuminata & Solitary & Elliptical & $23 \times 95$ & 0 & 0 & 5 & 4 & 0 & 0 & 0 & 0 & 0 & 0 \\
\hline 105 & Pinnularia tabellaria & Solitary & Elliptical & $16 \times 85$ & 0 & 0 & 0 & 1 & 0 & 0 & 0 & 0 & 3 & 0 \\
\hline 106 & Cyclotella comensis & Solitary & Round & $8.5 \times 11$ & 0 & 5 & 0 & 0 & 0 & 0 & 0 & 0 & 0 & 0 \\
\hline 107 & Cyclotella comta & Solitary & Drum & $10 \times 18$ & 0 & 4 & 0 & 0 & 0 & 0 & 1 & 0 & 0 & 0 \\
\hline 108 & Cyclotella stelligera & Colonial & Round & $8.0 \times 12$ & 0 & 0 & 1 & 0 & 0 & 0 & 2 & 0 & 0 & 0 \\
\hline 109 & Gonyostomum semen & Solitary & Oval & $28 \times 48$ & 3 & 0 & 0 & 0 & 0 & 0 & 0 & 0 & 1 & 0 \\
\hline 110 & Synura uvella & Solitary & Spherical & $7 \times 8.5$ & 0 & 0 & 4 & 0 & 1 & 1 & 0 & 0 & 0 & 0 \\
\hline
\end{tabular}

Table 3 showed the nine diversity indices of phytoplankton found in the 10 stations of Barishal City. In case of dominance index, the highest value was found in Station 8 and 9 (0.08) and the least in Station 2 (0.04). In terms of Simpson index, it was ranges from 0.92 to 0.96 among the all stations. Station 2 showed highest value by Shannon index and Equitability index, while Shannon index was lowest in Station 8 and equitability index was in Station 4,6 and 8. Pielou's index is a measure of diversity that quantifies how equal the community is numerically, and the value was highest for the Station 2 and 9 (0.77), while it was lowest in Station 4 (0.65). Menhinick's index was low (2.32) in Station7 and high in Station 9 (3.54). Similarly Margalef's index showed higher value in Station 2 (7.41) and lower value in Station 8 (5.12). Moreover, Fisher's alpha index and Berger- Parker index was highest in Station 9, but lowest in Station 7 and 2 respectively.

\section{DISCUSSION}

The Barishal City has numerous freshwater reservoirs but for the survey this experiment selected 10 reservoirs as sampling stations which were relatively old and large. And the stations demonstrated a rich number of phytoplanktons throughout the investigation. In terms of species number and percentage, the occurrence of Chlorophytes was dominant followed by Euglenophytes, Heterokontophytes and Cyanophytes, which indicated this group of green algae was common in this City (Figure 2). On the other hand, among the families the highest richness was represented by Euglenaceae (32 Taxa) 
Table 2: Position of each taxon in the taxonomic classification

\begin{tabular}{|c|c|c|c|c|c|}
\hline Domain & Phylum & Class & Order & Family & Taxa \\
\hline \multirow[t]{4}{*}{ Prokaryotes } & \multirow[t]{4}{*}{ Cyanophyta } & \multirow[t]{4}{*}{ Cyanophyceae } & Chroococcales & Chroococcaceae & $1-11$ \\
\hline & & & Nostocales & Nostocaceae & $12-13$ \\
\hline & & & Oscillatoriales & Rivulariaceae & 14 \\
\hline & & & & Oscillatoriaceae & $15-16$ \\
\hline \multirow[t]{20}{*}{ Eukaryotes } & \multirow[t]{12}{*}{ Chlorophyta } & \multirow[t]{3}{*}{ Charophyceae } & Charales & Characiaceae & $17-18$ \\
\hline & & & Desmidiales & Closteriaceae & $19-24$ \\
\hline & & & Zygnematales & Zygnemetaceae & $25-30$ \\
\hline & & \multirow[t]{9}{*}{ Chlorophyceae } & Chaetopeltidales & Chaetopeltidaceae & 31 \\
\hline & & & \multirow[t]{2}{*}{ Chlorellales } & Chlorellaceae & $32-36$ \\
\hline & & & & Oocystaceae & $37-40$ \\
\hline & & & \multirow[t]{3}{*}{ Sphaeropleales } & Hydrodictyaceae & 41 \\
\hline & & & & Scenedesmaceae & $42-50$ \\
\hline & & & & Selenastraceae & $51-53$ \\
\hline & & & Tetrasporales & Palmellaceae & $54-55$ \\
\hline & & & \multirow[t]{2}{*}{ Volvocales } & Chlamydomonadaceae & $56-59$ \\
\hline & & & & Volvocaceae & $60-61$ \\
\hline & Euglenophyta & Euglenophyceae & Euglenales & Euglenaceae & $62-93$ \\
\hline & \multirow{7}{*}{$\begin{array}{l}\text { Heterokonto- } \\
\text { phyta }\end{array}$} & \multirow[t]{5}{*}{ Bacillariophyceae } & Biddulphiales & Melosiraceae & $94-95$ \\
\hline & & & Cymbellales & Gomphonemataceae & $96-97$ \\
\hline & & & \multirow[t]{2}{*}{ Pinnales } & Bacillariaceae & $98-99$ \\
\hline & & & & Naviculaceae & $100-105$ \\
\hline & & & Thalassiosirales & Stephanodiscaceae & $106-108$ \\
\hline & & Raphidophyceae & Chattonellales & Raphidophyaceae & 109 \\
\hline & & Synurophyceae & Synurales & Synuraceae & 110 \\
\hline
\end{tabular}

Table 3: Diversity indices of phytoplankton of Barishal City during the study period among the 10 stations

\begin{tabular}{|c|c|c|c|c|c|c|c|c|c|c|}
\hline Indices & St.1 & St.2 & St.3 & St.4 & St.5 & St.6 & St.7 & St.8 & St.9 & St.10 \\
\hline Taxa_S & 31 & 37 & 36 & 36 & 28 & 30 & 27 & 24 & 24 & 37 \\
\hline Individuals & 110 & 129 & 126 & 118 & 89 & 131 & 136 & 89 & 46 & 152 \\
\hline Dominance index & 0.06 & 0.04 & 0.05 & 0.06 & 0.06 & 0.07 & 0.06 & 0.08 & 0.08 & 0.05 \\
\hline Simpson index & 0.94 & 0.96 & 0.95 & 0.94 & 0.94 & 0.93 & 0.94 & 0.92 & 0.92 & 0.95 \\
\hline Shannon index & 3.06 & 3.35 & 3.25 & 3.15 & 3.02 & 2.99 & 2.99 & 2.80 & 2.91 & 3.28 \\
\hline Pielou's index & 0.69 & 0.77 & 0.72 & 0.65 & 0.73 & 0.66 & 0.74 & 0.69 & 0.77 & 0.72 \\
\hline Menhinick's index & 2.96 & 3.26 & 3.20 & 3.31 & 2.97 & 2.62 & 2.32 & 2.54 & 3.54 & 3.00 \\
\hline Margalef's index & 6.38 & 7.41 & 7.24 & 7.34 & 6.02 & 5.95 & 5.30 & 5.12 & 6.00 & 7.17 \\
\hline Equitability index & 0.89 & 0.93 & 0.91 & 0.88 & 0.91 & 0.88 & 0.91 & 0.88 & 0.92 & 0.91 \\
\hline Fisher's alpha index & 14.36 & 17.35 & 16.84 & 17.65 & 14.05 & 12.17 & 10.11 & 10.79 & 20.25 & 15.57 \\
\hline Berger-Parker index & 0.16 & 0.09 & 0.10 & 0.15 & 0.10 & 0.13 & 0.12 & 0.16 & 0.20 & 0.10 \\
\hline
\end{tabular}

followed by Chroococcaceae (11 Taxa), Scenedesmaceae (9 Taxa), Zygnemetaceae (6 Taxa), Closteriaceae (6 Taxa) and Chlorellaceae (5 Taxa). Among all taxa, Scenedesmus was mostly frequent genus in Chlorophyta, while Merismopedia in Cyanophyta, Euglena in Euglenophyta, and Navilcula, Pinnularia, Cyclotella were in Heterokontopyta. Furthermore, as a single genus, Euglena possessed the highest number of taxa (13) throughout the survey, while the 27 genera reported with only single species.

Diversity measurements have many potential applications in any aquatic ecosystems as part of the ecological study. In this investigation, Station 8 and 9 indicates the more dominancy by species number than the other stations. And, the species abundance was found highest in the Station 2 according to Simpson and Shannon diversity index. As the evenness or Pielou's index means how equal the community is numerically in an ecosystem, Station 2 and 9 showed greater results over the others. According to Menhinick's index, Fisher alpha index and Berger- Parker index, the
Station 9 demonstrated the highest richness of species. On the other hand, Station 2 demonstrated the best species richness according to Margalef's index and Equitability index. However, assessing the nine diversity indices, the diversity of the listed phytoplanktons was more prominent in Station 2 and 9, whereas Station 8 earned lowest marks in five indices out of the nine.

In terms of species distribution through the stations, 56 taxa were found common regardless of rare and abundant frequency in several stations. On the other hand, 12 species were found only abundantly and 42 taxa were found rarely in some of the stations. The species Teilingia exigua, Chlorella vulgaris, Planktosphaeria gelatinosa, Kirchneriella contorta, Kirchneriella irregularis, Korshikoviella limnetica and Euglena granulata were found rarely only in Station 3, 10, 5, 7, 1, 4 and 9 respectively (Figure 3). On the other hand, the appearance of Gloeothece rupestris, Microcystis aeruginosa, Microcystis flosaquae, Oscillatoria formosa and Scenedesmus acuminatus were common in maximum stations (Figure 3). 


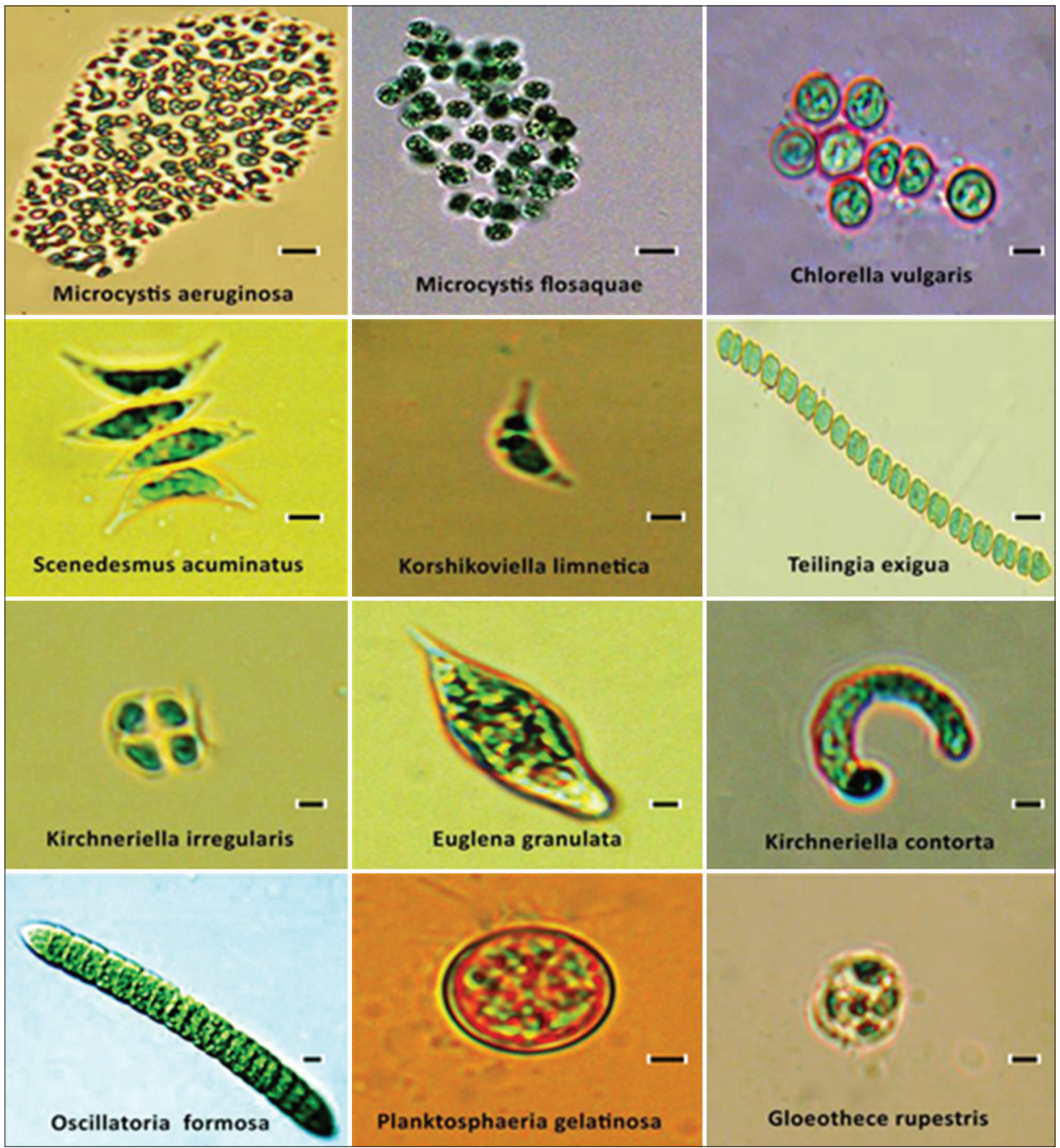

Figure 3: The rarest and the most common phytoplanktons of Barishal City. Bar $=1 \mu \mathrm{m}$

\section{CONCLUSIONS}

There was no new species report and all listed taxa were reported previously from different locations of Bangladesh by different authors. However, this is the first report on phytoplanktons community from the Barishal City of Bangladesh.

\section{REFERENCES}

1. Andersen RA. Algal culturing techniques, Elsevier Academic Press, London. 2005; 83-85.

2. Chiara L, Andersen JH., Espen H, Marte A, Laura E, Francesco E, Kirsti H, Hanssen KØ, Giovanna R, Adrianna I. Bioactivity screening of microalgae for antioxidant, anti-Inflammatory, anticancer, antidiabetes, and antibacterial activities. Frontiers in Marine Science. 2016; 3: 68 .

3. Thajuddin N and Dhanasekaran D. Algae- organisms for imminent biotechnology, London. 2016; 237-76.

4. Ramaraj $\mathrm{S}$ et al. Microalgae as an attractive source for biofuel production (Thangavel P and Sridevi G Eds.), Environmental sustainability. New Delhi. 2015; 129.

5. Banglapedia- the national encyclopedia of Bangladesh. http:// en. banglapedia.org/index. php?title=Barisal_City_Corporation
6. Khondker M, Bhuiyan R, Yeasmin J., et al. New records of phytoplankton for Bangladesh. 2. Cryptophyceae and Synurophyceae. Bangladesh Journal of Botany. 2007; 36: 53-59.

7. Khondker M, Bhuiyan R, Yeasmin, J, et al. New records of phytoplankton for Bangladesh. 2. Cryptophyceae and Synurophyceae. Bangladesh Journal of Botany. 2006; 35: 53-59.

8. Bellinger EG and Sigee DC. Freshwater algae: identification and use as bioindicators, John Wiley \& Sons, USA. 2010; 244.

9. Ahmed ZU, Khondker M, Begum ZNT, et al. Encyclopedia of flora and fauna of Bangladesh, Asiatic Soc. Bangladesh, Dhaka. 2009; 543

10. Islam AKMN and Alfasane MA. Euglenophyceae from Barisal district, Bangladesh: III. Genus Trachelomonas Ehr. Bangladesh Journal of Plant Taxonomy. 2004; 11: 33-37.

11. Islam AKMN and Moniruzzaman K. Contribution to the study on Euglenophyta of Bangladesh. I. Genus Trachelomonas Ehr. Internationale Revue der gesamten Hydrobiologie. 1981; 66: 109-125.

12. Smith GM. Freshwater algae of the United States, New York 1950; 719

13. Lee RE. Phycology, Cambridge University Press, New York. 2008; 561

14. Komárek J and Fott B. Chlorococcales (Huber-Pestalozzi, Eds.), Das Phytoplankton des Süsswassers, Systematik u. Biologie, Teil 1 , Stuttgart. 1983; 1044.

15. Bold HC and Wynne MJ. Introduction to the Algae, Prentice-Hall, New Jersey. 1985; 706 